\title{
Eficacia clínica del MTA en Pulpotomías de pacientes pediátricos: Una Revisión Sistemática
}

\author{
Sol Gabriela Holguín García, ${ }^{1}$
}

\section{Resumen}

Objetivo: Analizar la eficacia clínica del Agregado Trióxido Mineral (MTA) en pulpotomías a través de estudios comparativos con el formocresol, sulfato férrico e hidróxido de calcio; en pacientes pediátricos con edad de tres a nueve años. Materiales y Métodos: El artículo se basa en una revisión sistemática de la literatura, por ello, se utilizaron como fuentes de información las bases de datos: PubMed, Scielo, y Biblioteca Virtual de Salud. Asimismo, como criterios de inclusión se establecieron publicaciones del año 2008 al 2019, cuya procedencia se derivó de journals y revistas académicas arbitradas; los cuales permitieron obtener dieciséis ensayos que evaluaban la eficacia clínica a través de los signos y síntomas de las patologías pulpares más comunes: absceso, inflamación gingival, movilidad patológica, dolor a la percusión y dolor espontáneo. Resultados: El MTA obtuvo una eficacia de $97,9 \%$ en contraposición al $86,9 \%$ del Sulfato Férrico. En relación al Formocresol, el MTA logró el 99\% contra un $98,3 \%$ de eficacia. Además, en la comparación del MTA con el Hidróxido de Calcio el primero logró un $98,2 \%$ y el segundo $74,5 \%$. Conclusiones: El MTA obtuvo una mayor eficacia clínica debido a su biocompatibilidad, $\mathrm{pH}$ básico y su elevada facultad al sellar la cámara pulpar. Por lo tanto, puede emplearse como un material seguro para las pulpotomías de pacientes pediátricos.

Palabras Clave: MTA, pulpotomía, eficacia clínica. 
Artigo da revisao

\section{Eficácia Clínica da MTA em Pulpotomias Pediátricas de Pacientes: Uma Revisão Sistemática}

\section{Resumo}

Objetivo: Analisar a eficácia clínica do Agregado de Trióxido Mineral (MTA) em pulpotomias através de estudos comparativos com formocresol, sulfato férrico e hidróxido de cálcio; em pacientes pediátricos de três a nove anos de idade. Materiais e Métodos: $\mathrm{O}$ artigo é baseado em uma revisão sistemática da literatura, portanto, bases de dados foram utilizadas como fontes de informação: PubMed, Scielo, e Biblioteca Virtual. Da mesma forma, como critério de inclusão, foram estabelecidas publicações de 2008 a 2019, cuja origem foi derivada de periódicos e revistas acadêmicas de referência; o que nos permitiu obter dezesseis ensaios que avaliaram a eficácia clínica através dos sinais e sintomas das doenças mais comuns da polpa: abscesso, inflamação gengival, mobilidade patológica, dor na percussão e dor espontânea. Resultados: A MTA obteve uma eficácia de 97,9\% contra $86,9 \%$ para o sulfato férrico. Em relação ao Formocresol, o MTA alcançou $99 \%$ contra 98,3\% de eficácia. Além disso, na comparação do MTA com o hidróxido de cálcio, o primeiro atingiu $98,2 \%$ e o segundo $74,5 \%$. Conclusões: A MTA obteve uma maior eficácia clínica devido a sua biocompatibilidade, $\mathrm{pH}$ básico e sua alta faculdade ao selar a câmara de celulose. Portanto, pode ser usado como material segura para pulpotomias de pacientes pediátricos.

Palavras-chave: MTA, pulpotomia, eficácia clínica

Article of revision

\section{Clinical Efficacy of MTA in Pediatric Patient Pulpotomies: A Systematic Review}

\begin{abstract}
Objective: To analyze the clinical efficacy of the Mineral Trioxide Aggregate (MTA) in pulpotomies through comparative studies with formocresol, ferric sulfate and calcium hydroxide; in pediatric patients aged three to nine years. Materials and Methods: The article is based on a systematic review of the literature, therefore, databases were used as sources of information: PubMed, Scielo, and Virtual Health Library. Likewise, as inclusion criteria, publications from 2008
\end{abstract}

to 2019 were established, whose origin was derived from journals and refereed academic journals; which allowed us to obtain sixteen trials that evaluated clinical efficacy through the signs and symptoms of the most common pulp diseases: abscess, gingival inflammation, pathological mobility, pain on percussion and spontaneous pain. Results: MTA obtained an efficacy of $97.9 \%$ as opposed to $86.9 \%$ for ferric sulfate. Concerning to Formocresol, MTA achieved $99 \%$ versus $98.3 \%$ efficacy. Also, in the comparison of MTA with Calcium Hydroxide, the 
former achieved $98.2 \%$ and the latter 74.5\%. Conclusions: MTA obtained a higher clinical efficacy due to its biocompatibility, basic $\mathrm{pH}$ and its high faculty when sealing the pulp chamber. Therefore, it can be used as a safe

\section{Introducción}

Odabas et al. ${ }^{1}$ señalan que el formocresol es el material más utilizado en el procedimiento de pulpotomía, porque al ser momificante realiza la desnaturalización de las proteínas de la pulpa radicular extendiéndose hacia la pulpa más apical logrando fijar los tejidos; sin embargo, uno de sus principales componentes activos es el formaldehido. En este ámbito, la International Agency for Research on Cancer (IARC) ${ }^{2}$ evidencia que el vapor de formaldehído es un carcinógeno para los humanos.

Por su parte, Zaror et $a .^{3}$ manifiestan que el glutaraldehído, electrocirugía, laser, hidróxido de calcio, proteínas morfogenéticas e hipoclorito de sodio han sido propuestos como opciones para las pulpotomías, pero los resultados obtenidos han sido diversos. No obstante, el sulfato férrico y el MTA han demostrado mayor eficacia clínica incluso similar al formocresol. ${ }^{3}$

En este contexto, Srinivasan et al. ${ }^{4}$ indican que el sulfato férrico es utilizado como agente hemostático en pulpotomías de dientes primarios. Asimismo, Assed et al..$^{5}$ señalan que su uso puede prevenir los problemas originados con la formación del coágulo después de la remoción de la cámara pulpar. En cambio, Biedma et al. ${ }^{6}$ determinan que al no tener la capacidad material for pediatric patient pulpotomies.

Key words: MTA, pulpotomy, clinical efficacy.

de formar una nueva dentina es posible la presencia de reabsorción interna.

Según Valdivieso et al.7, el hidróxido de calcio desde el punto de vista biológico se considera eficaz en pulpotomías, porque no solo mantiene la vitalidad pulpar, también, favorece la formación de un puente dentinario que es similar a la osteodentina.

Por otra parte, Rodríguez et al. ${ }^{8}$ consideran que el MTA no es un material tóxico, es biocompatible con los tejidos, no es mutagénico, tiene radiopacidad, es de rápida eliminación cuando existen excedentes, tiene propiedades hidrofílicas y es de fácil manipulación; particularidades que propician su eficacia. Además, Aguado et al. ${ }^{9}$ establecen que el MTA no permite el desarrollo de bacterias ni hongos; lo cual se correlaciona con lo señalado por Srinivasan et $a .^{4}{ }^{4}$, que resaltan la alta capacidad del MTA para resistir la penetración de microrganismos que podrían persistir o presentarse por filtración coronal incluso después de eliminar la caries o al realizar el desbridamiento del conducto radicular.

Por consecuencia, en la actualidad se realizan investigaciones sobre materiales para terapias pulpares que estén direccionadas a desarrollar biomateriales que estimulen el proceso de regeneración de la dentina; ${ }^{10}$ y según Caicedo et al. ${ }^{11}$ no 
solo debe evaluarse la biocompatibilidad entre la interacción del material y el tejido, sino también valorar el potencial para obtener una respuesta sobre el tejido intervenido. En este ámbito, Biedma et al. ${ }^{6}$ señalan que dentro del grupo de materiales regenerativos para pulpotomías en dientes primarios consta el MTA.

Por los motivos antes expuestos, este artículo tiene como objetivo analizar la eficacia clínica del MTA en pulpotomías de pacientes pediátricos, por lo cual, se realizará una revisión sistemática de estudios comparativos entre el MTA y sus homólogos: formocresol, sulfato férrico e hidróxido de calcio; en niños con un rango de edad de tres a nueve años.

\section{Materiales y métodos}

La Revisión Sistemática permite identificar, evaluar, interpretar y sintetizar investigaciones existentes o relevantes ${ }^{12}$, por consecuencia para su realización se siguió la estructura formulada por Kitchenham $^{13}$ y se complementó la parte conceptual con el manual descrito por Higgins et al. ${ }^{12}$

El protocolo utilizado se enfoca principalmente en seis aspectos: i) Pregunta de Investigación, ii) Selección de Fuentes, Definición de Cadenas de Búsqueda y Criterios de Selección, iii) Selección de Estudios: Ejecución de Procedimiento, iv) Aplicación de Criterios de Calidad, v) Extracción de Datos, y vi) Reporte de Resultados. En la Figura 1 se esquematiza el protocolo empleado.

\section{Pregunta de Investigación}

Una pregunta de investigación establece los criterios de elegibilidad, búsqueda de estudios, recolección de los datos de estudios seleccionados, y presentación de los resultados. ${ }^{12}$ Por tal motivo, para su formulación se empleó el método PICO (Participantes, Intervenciones, Comparaciones y Outcome (Desenlaces)) debido a que permite especificar el tipo de población, clase de intervenciones y tipo desenlace que será de interés. ${ }^{12} \mathrm{La}$ pregunta planteada es la siguiente:

RQ1: ¿Cuál es el porcentaje de eficacia clínica del MTA en pulpotomías en contraposición al formocresol, hidróxido de calcio y el sulfato férrico en pacientes pediátricos de tres a nueve años de edad?

Selección de Fuente, Definición de Cadenas de Búsqueda y Criterios de Selección

Uno los elementos fundamentales para la revisión sistemática es realizar una búsqueda que identifique todos los estudios que puedan cumplir los criterios de elegibilidad, esto garantizará que sea amplia, objetiva y reproducible ${ }^{12}$. Partiendo de la condición anterior se tomaron como fuentes potenciales de información cuatro bases de datos: i) PubMed, ii) Scielo, iii) Biblioteca Virtual de Salud, y iv) se tomó especial atención a la revista especializada en endodoncia de mayor impacto: International Endodontic Journal; cabe señalar que en el momento de realizar la exploración estratégica se utilizó la opción de Búsqueda Avanzada.

Por otra parte, se usaron como criterios de inclusión: publicaciones del año 2008 al 2019, documentos tipo: revistas científicas (journals), publicaciones de conferencias (conference publications) y publicaciones académicas arbitradas; y como tipos de estudio: ensayos clínicos en inglés, portugués y español. Además, los criterios 


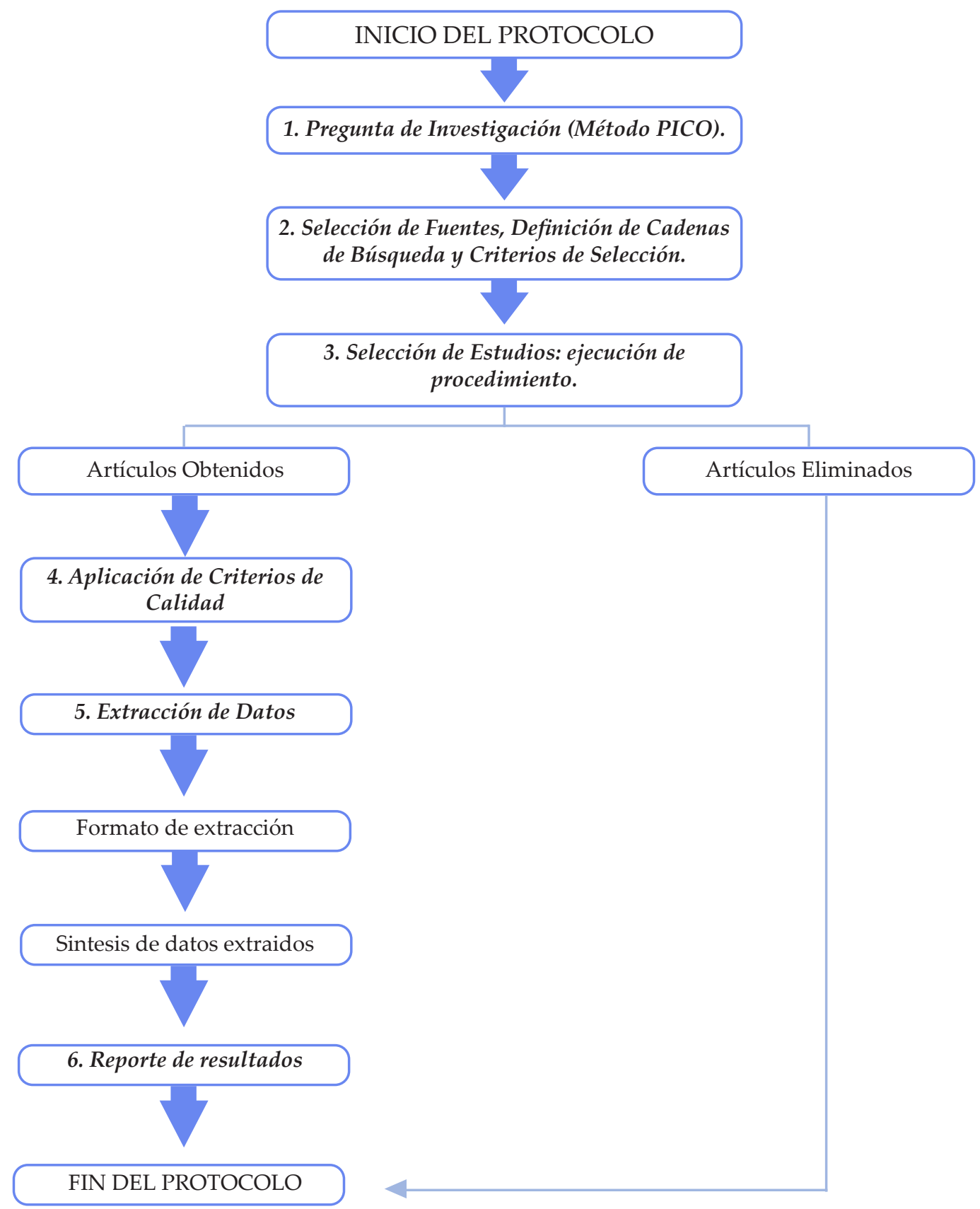

Figura 1: Diagrama del Protocolo de Revisión Sistemática.

Fuente: Kitchenham. Procedures for Performing Systematic Reviews, Keele University Technical Report. 2004

de exclusión fueron: documentos sin resultados experimentales y documentos de literatura secundaria.

En este contexto, las cadenas de búsqueda se generaron combinando las palabras claves de la pregunta de investigación con los conectores lógicos " $A N D$ " y "OR", asimismo, se utilizaron sinónimos. Es necesario acotar que las palabras claves y derivadas también se consideraron en idioma inglés, porque en dicha lengua se presentan mayor número de investigaciones. En la Tabla 1 se detallan los parámetros de búsqueda empleados. 
Tabla 1. Parámetros utilizados en el proceso de búsqueda de la investigación

\begin{tabular}{|c|c|c|}
\hline Base de Datos & Palabras Claves & Cadena de Búsqueda Aplicada \\
\hline $\begin{array}{l}\text { PubMed } \\
\text { https://www.ncbi.nlm.nih.gov/pubmed }\end{array}$ & $\begin{array}{l}\text { Pulpotomía } \\
\text { Dientes primarios. } \\
\text { MTA }\end{array}$ & $\begin{array}{l}\text { ("pulpotomy"[MeSH Terms] OR "pul- } \\
\text { potomy"[All Fields]) AND primary[All } \\
\text { Fields] }\end{array}$ \\
\hline $\begin{array}{l}\text { Scielo } \\
\text { http://www.scielo.org }\end{array}$ & Eficacia clínica & $\begin{array}{l}\text { tw:(mta en pulpotomía ) AND (instan- } \\
\text { ce:"regional") }\end{array}$ \\
\hline $\begin{array}{l}\text { Biblioteca Virtual De Salud } \\
\text { https://bvsalud.org }\end{array}$ & $\begin{array}{l}\text { Formocresol } \\
\text { Hidróxido de calcio }\end{array}$ & $\begin{array}{l}\text { ("pulpotomy"[MeSH Terms] OR "pul- } \\
\text { potomy"[All Fields]) AND primary[All }\end{array}$ \\
\hline $\begin{array}{l}\text { International Endodontic Journal } \\
\text { https://onlinelibrary.wiley.com }\end{array}$ & $\begin{array}{l}\text { Sulfato Férrico } \\
\text { Niños de } 3 \text { a } 9 \text { años }\end{array}$ & $\begin{array}{l}\text { Fields] AND mta[All Fields] AND ("for- } \\
\text { mocresol"[Supplementary Concept] OR } \\
\text { "formocresol"[All Fields]) } \\
\text { ("pulpotomy"[MeSH Terms] OR "pul- } \\
\text { potomy"[All Fields]) AND ("child"[- } \\
\text { MeSH Terms] OR "child"[All Fields] } \\
\text { OR "children"[All Fields]) AND 3[All } \\
\text { Fields] AND 9[All Fields] AND ("Age"[- } \\
\text { Journal] OR "Age (Omaha)"[Journal] OR } \\
\text { "Age (Dordr)"[Journal] OR "Adv Genet } \\
\text { Eng"[Journal] OR "age"[All Fields]) }\end{array}$ \\
\hline
\end{tabular}

Selección de Estudios: Ejecución de Procedimientos

El procedimiento consta de los siguientes pasos:

a. Con base a los parámetros definidos en Tabla 1 se inicia la búsqueda de los artículos y luego se procede a guardarlos utilizando el programa Zotero.

b. Eliminación de artículos duplicados a través del programa Zotero.

c. Exclusión de los artículos resultantes del paso anterior cuyos títulos o palabras claves no estaban directamente relacionados con la pregunta de investigación.

d. Supresión de los artículos obtenidos del paso anterior, cuyo contenido no guarda relación directa con la pregunta de investigación.

En la Tabla 2 se evidencian los resultados obtenidos.

\section{Aplicación de Criterios de Calidad}

Los criterios de calidad empleados están basados del "Quality Assessment" propuestos por Kitchenham et al. ${ }^{14}$, en donde se utilizan cuatro preguntas para evaluar la calidad de un estudio. En la Tabla 3 se explican los criterios utilizados.

En la Tabla 4 se exponen los resultados obtenidos en esta investigación aplicando los Criterios de Calidad expuestos en la Tabla 3. 
Tabla 2: Resultados de la ejecución del procedimiento para la selección de estudios

\begin{tabular}{|l|c|c|c|c|c|}
\hline Base de Datos & Fecha de Extracción & Paso 1 & Paso 2 & Paso 3 & Paso 4 \\
\hline PubMed & $02-06-2019$ & 607 & 512 & 452 & 7 \\
\hline Scielo & $02-06-2019$ & 112 & 91 & 86 & 3 \\
\hline Biblioteca Virtual de salud & $02-06-2019$ & 44 & 42 & 35 & 4 \\
\hline International Endodontic Journal & $02-06-2019$ & 9 & 9 & 6 & 2 \\
\hline \multicolumn{7}{|c|}{ Total de Artículos Seleccionados } & 16 \\
\hline
\end{tabular}

Tabla 3: Criterios de Calidad

\begin{tabular}{|c|c|c|c|}
\hline Identificación & Pregunta & Evaluación & Puntaje \\
\hline EQ1 & $\begin{array}{l}\text { ¿Se describen apropiadamente } \\
\text { los criterios de inclusión y ex- } \\
\text { clusión de la Investigación? }\end{array}$ & $\begin{array}{l}\mathrm{Y}=\mathrm{si} \\
\mathrm{N}=\text { no } \\
\mathrm{P}=\text { parcialmente }\end{array}$ & $\begin{array}{l}\mathrm{Y}=1 \\
\mathrm{~N}=0 \\
\mathrm{P}=0,5\end{array}$ \\
\hline EQ2 & $\begin{array}{l}\text { ¿Es probable que la investiga- } \\
\text { ción bibliográfica cubra todos } \\
\text { los estudios pertinentes? }\end{array}$ & $\begin{array}{l}\text { Los autores han buscado artículos: } \\
\mathrm{Y}=>=4+\text { adicional estrategia } \\
\mathrm{N}=3 \text { o } 4 \text { sin extra } \\
\mathrm{P}=2 \text { o conjunto restringido de revista. }\end{array}$ & $\begin{array}{l}Y=1 \\
N=0 \\
P=0,5\end{array}$ \\
\hline EQ3 & $\begin{array}{l}\text { ¿Los evaluadores evaluaron la } \\
\text { calidad/validez de los estudios } \\
\text { incluidos? }\end{array}$ & $\begin{array}{l}\mathrm{Y}=\text { Los autores definieron explícitamente } \\
\text { los criterios calidad. } \\
\mathrm{N}=\text { no es explicita. } \\
\mathrm{P}=\text { la pregunta de investigación involucra } \\
\text { cuestiones de calidad. }\end{array}$ & $\begin{array}{l}Y=1 \\
N=0 \\
P=0,5\end{array}$ \\
\hline EQ4 & $\begin{array}{l}\text { ¿Fueron adecuadamente descri- } \\
\text { tos los datos/estudios básicos? }\end{array}$ & $\begin{array}{l}\mathrm{Y}=\text { información sobre cada estudio. } \\
\mathrm{N}=\text { no especifica resultados de estudios } \\
\text { primarios individuales. } \\
\mathrm{P}=\text { información resumida de estudios } \\
\text { primarios. }\end{array}$ & $\begin{array}{l}Y=1 \\
N=0 \\
P=0,5\end{array}$ \\
\hline
\end{tabular}

Fuente: Kitchenham, et al., Systematic literature reviews in software engineering-A systematic literature review. Information and Software Technology. 2009 
Tabla 4: Resultados de la Aplicación de Criterios de Calidad

\begin{tabular}{|c|c|c|c|c|c|c|c|c|}
\hline Artículo & 交 & $\stackrel{n}{\pi}$ & 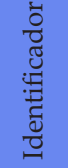 & $\overline{\mathrm{O}}$ & Оี & Oి & 䓑 & $\begin{array}{l}\frac{0}{\pi} \\
\Xi \\
\Xi \\
\Xi\end{array}$ \\
\hline $\begin{array}{l}\text { Pulpotomías con sulfato férrico y MTA en dientes primarios: serie } \\
\text { de casos. }\end{array}$ & 2011 & Chile & S1 & $\mathrm{Y}$ & $\mathrm{Y}$ & Y & Y & 4 \\
\hline $\begin{array}{l}\text { Clinical, Radiographic and Histological Evaluation of Primary Teeth } \\
\text { Pulpotomy Using MTA And Ferric Sulfate. }\end{array}$ & 2018 & Brazil & S2 & $\mathrm{Y}$ & Y & Y & Y & 4 \\
\hline $\begin{array}{l}\text { Success rates of pulpotomies in primary molars using calcium Silica- } \\
\text { te-based materials: a randomized control trial. }\end{array}$ & 2017 & Turquía & S3 & $\mathrm{Y}$ & $\mathrm{Y}$ & $\mathrm{P}$ & $Y$ & 3.5 \\
\hline $\begin{array}{l}\text { Clinical and radiographic comparison of various medicaments used } \\
\text { for pulpotomy in primary molars: a randomized clinical trial. }\end{array}$ & 2016 & India & S4 & $\mathrm{Y}$ & Y & Y & Y & 4 \\
\hline $\begin{array}{l}\text { Pulpotomías en molares primarios evaluación clínica, radiográfica } \\
\text { de formocresol o trióxido mineral agregado. }\end{array}$ & 2008 & Argentina & S5 & $\mathrm{Y}$ & $\mathrm{Y}$ & Y & Y & 4 \\
\hline $\begin{array}{l}\text { Comparison of mineral trioxide aggregate and formocresol as pulp } \\
\text { medicaments for pulpotomies in primary molars. }\end{array}$ & 2008 & Irán & S6 & $\mathrm{Y}$ & $\mathrm{Y}$ & Y & $Y$ & 4 \\
\hline $\begin{array}{l}\text { Comparative investigation of clinical/radiographical signs of mineral } \\
\text { trioxide aggregate and formocresol on pulpotomized primary } \\
\text { molars. }\end{array}$ & 2010 & India & S7 & $\mathrm{Y}$ & $\mathrm{Y}$ & Y & Y & 4 \\
\hline $\begin{array}{l}\text { Mineral trioxide aggregate and formocresol pulpotomy of primary } \\
\text { teeth: a 2-year follow- up. }\end{array}$ & 2010 & Irán & S8 & $\mathrm{Y}$ & $\mathrm{Y}$ & Y & Y & 4 \\
\hline $\begin{array}{l}\text { Effects of mineral trioxide aggregate and formocresol on vital pulp } \\
\text { after pulpotomy of primary molars: an in vivo study. }\end{array}$ & 2011 & India & S9 & Y & $\mathrm{Y}$ & $\mathrm{P}$ & Y & 3,5 \\
\hline $\begin{array}{l}\text { In vivo comparative evaluation of mineral trioxide aggregate and } \\
\text { formocresol pulpotomy in primary molars: a } 60 \text {-month follow-up } \\
\text { study. }\end{array}$ & 2017 & India & S10 & $\mathrm{Y}$ & $\mathrm{Y}$ & Y & Y & 4 \\
\hline $\begin{array}{l}\text { Evaluation and comparison of white mineral trioxide aggregate and } \\
\text { formocresol medicaments in primary tooth pulpotomy: clinical and } \\
\text { radiographic study. }\end{array}$ & 2014 & India & S11 & $\mathrm{Y}$ & $\mathrm{Y}$ & $\mathrm{Y}$ & $P$ & 3,5 \\
\hline $\begin{array}{l}\text { Clinical and radiographic evaluation of the effectiveness of formo- } \\
\text { cresol, mineral trioxide aggregate, portland cement, and enamel ma- } \\
\text { trix derivative in primary teeth pulpotomies: a two-year follow-up. }\end{array}$ & 2016 & Turquía & S12 & $\mathrm{Y}$ & $\mathrm{Y}$ & $\mathrm{Y}$ & $Y$ & 4 \\
\hline $\begin{array}{l}\text { Clinical and radiographic evaluation of white MTA versus formocre- } \\
\text { sol Pulpotomy: a 48-month follow-up study. }\end{array}$ & 2017 & España & S13 & $\mathrm{Y}$ & $\mathrm{Y}$ & $\mathrm{Y}$ & $\mathrm{Y}$ & 4 \\
\hline $\begin{array}{l}\text { Effect of mineral trioxide aggregate and formocresol pulpotomy on } \\
\text { vital primary teeth: a clinical and radiographic study. }\end{array}$ & 2015 & Nigeria & S14 & Y & $\mathrm{Y}$ & Y & Y & 4 \\
\hline $\begin{array}{l}\text { Evaluation of formocresol, calcium hydroxide, ferric sulfate, and } \\
\text { MTA primary molar pulpotomies. }\end{array}$ & 2014 & Turquía & S15 & $\mathrm{Y}$ & $\mathrm{Y}$ & $\mathrm{Y}$ & $\mathrm{Y}$ & 4 \\
\hline $\begin{array}{l}\text { The effectiveness of mineral trioxide aggregate, calcium hydroxide } \\
\text { and formocresol for pulpotomies in primary teeth. }\end{array}$ & 2008 & Brazil & S16 & $\mathrm{Y}$ & $\mathrm{Y}$ & $\mathrm{P}$ & $\mathrm{Y}$ & 3,5 \\
\hline
\end{tabular}

\section{Resultados}

\section{Extracción de Datos}

Durante esta fase se determinó de los artículos seleccionados la siguiente información: autor, tipo de investigación, muestra, edad de los pacientes, pulpotomías realizadas, hallazgos clínicos y eficacia clínica. Además, la selección de los hallazgos clínicos se basa en los signos y síntomas de patologías pulpares más comunes en la respuesta pulpar, los cuales son: absceso, inflamación gingival, movilidad patológica, dolor a la percusión y dolor espontáneo. ${ }^{6}$ 
Por otra parte, para registrar los resultados obtenidos se utilizó la aplicación Microsoft Excel. Esta herramienta permitirá en un momento posterior comparar y analizar los artículos de una forma más eficiente; de tal manera que se obtenga la respuesta a la pregunta de investigación planteada. A continuación, en las Tablas 5, 6 y 7, se detallan los resultados alcanzados.

Tabla 5: Resultados de Eficacia Clínica entre el MTA y el Sulfato Férrico obtenidos en la Extracción de Datos.

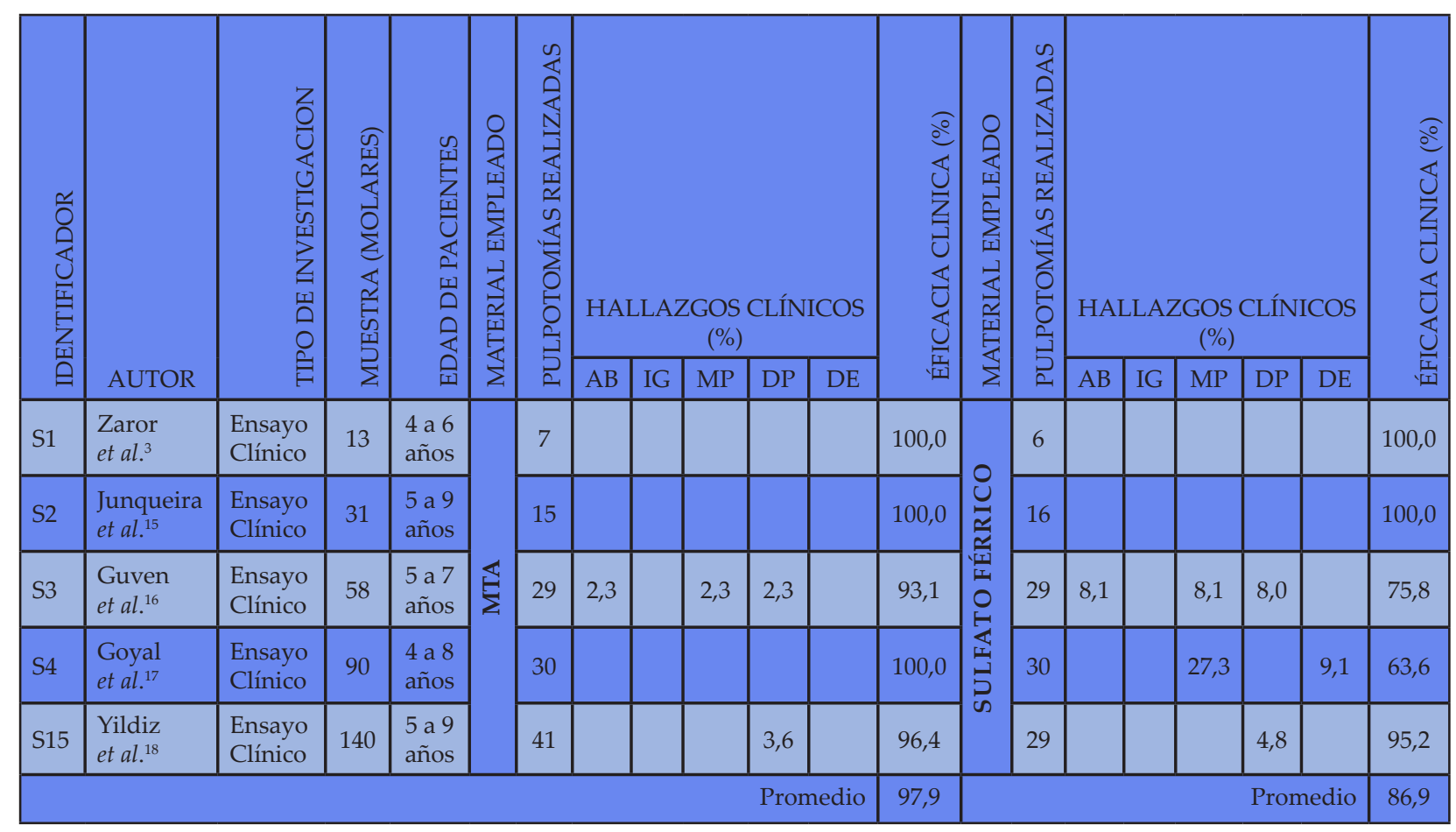

Leyenda

\begin{tabular}{|l|l|l|}
\hline Hallazgos & Sigla & \multicolumn{1}{|c|}{ Significado } \\
\hline \multirow{4}{*}{ Clínicos } & $\mathrm{AB}$ & Absceso \\
\cline { 2 - 3 } & $\mathrm{IG}$ & Inflamación Gingival \\
\cline { 2 - 3 } & $\mathrm{MP}$ & Movilidad Patológica \\
\cline { 2 - 3 } & $\mathrm{DP}$ & Dolor a la Percusión \\
\cline { 2 - 3 } & $\mathrm{DE}$ & Dolor Espontáneo \\
\hline
\end{tabular}


Tabla 6: Resultados de Eficacia Clínica entre el MTA y el Formocresol obtenidos en la Extracción de Datos

\begin{tabular}{|c|c|c|c|c|c|c|c|c|c|c|c|c|c|c|c|c|c|c|c|c|}
\hline \multirow[t]{2}{*}{ 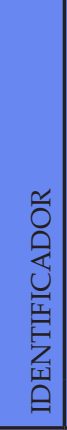 } & \multirow[b]{2}{*}{ AUTOR } & \multirow[t]{2}{*}{ 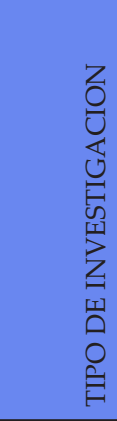 } & \multirow[t]{2}{*}{ 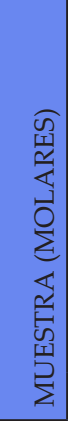 } & \multirow[t]{2}{*}{ 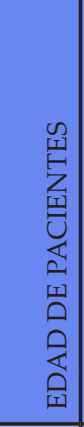 } & \multirow[t]{2}{*}{ 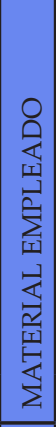 } & \multirow[t]{2}{*}{ 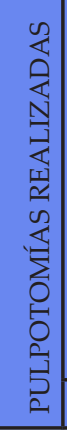 } & \multicolumn{5}{|c|}{$\begin{array}{l}\text { HALLAZGOS CLÍNICOS } \\
(\%)\end{array}$} & \multirow[t]{2}{*}{ 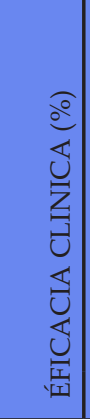 } & \multirow[t]{2}{*}{ 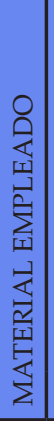 } & \multirow[t]{2}{*}{ 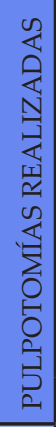 } & \multicolumn{5}{|c|}{$\begin{array}{c}\text { HALLAZGOS CLÍNICOS } \\
(\%)\end{array}$} & \multirow[t]{2}{*}{ 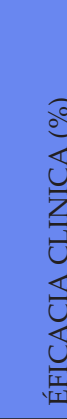 } \\
\hline & & & & & & & $\mathrm{AB}$ & IG & $\mathrm{MP}$ & DP & $\mathrm{DE}$ & & & & $\mathrm{AB}$ & IG & $\mathrm{MP}$ & DP & $\mathrm{DE}$ & \\
\hline S5 & $\begin{array}{l}\text { Biondi } \\
\text { et al. } .^{19}\end{array}$ & $\begin{array}{l}\text { Ensayo } \\
\text { Clínico }\end{array}$ & 30 & $\begin{array}{l}5 \text { a } 8 \\
\text { años }\end{array}$ & \multirow{11}{*}{$\overleftrightarrow{\xi}$} & 15 & & & 6,0 & & & 94,0 & \multirow{11}{*}{ 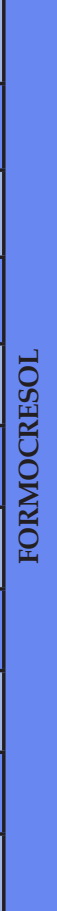 } & 15 & 6,0 & & & & & 94,0 \\
\hline S6 & $\begin{array}{l}\text { Nooro- } \\
\text { llahian }{ }^{20}\end{array}$ & $\begin{array}{l}\text { Ensayo } \\
\text { Clínico }\end{array}$ & 60 & $\begin{array}{l}5 \text { a } 7 \\
\text { años }\end{array}$ & & 30 & & & & & & 100,0 & & 30 & & & & & & 100,0 \\
\hline S7 & $\begin{array}{l}\text { Hugar } \\
\text { et al. } .^{21}\end{array}$ & $\begin{array}{l}\text { Ensayo } \\
\text { Clínico }\end{array}$ & 60 & $\begin{array}{l}5 \text { a } 8 \\
\text { años }\end{array}$ & & 30 & & & & & & 100,0 & & 30 & & & & & & 100,0 \\
\hline S8 & $\begin{array}{l}\text { Ansari } \\
\text { et al. } .^{22}\end{array}$ & $\begin{array}{l}\text { Ensayo } \\
\text { Clínico }\end{array}$ & 40 & $\begin{array}{l}4 \text { a } 9 \\
\text { años }\end{array}$ & & 20 & & & & & & 100,0 & & 20 & 1,2 & & & & & 98,8 \\
\hline S9 & $\begin{array}{l}\text { Godhi } \\
\text { et al. } .^{23}\end{array}$ & $\begin{array}{l}\text { Ensayo } \\
\text { Clínico }\end{array}$ & 50 & $\begin{array}{l}5 \text { a } 8 \\
\text { años }\end{array}$ & & 25 & & & & & & 100,0 & & 25 & & & & & & 100,0 \\
\hline S10 & $\begin{array}{l}\text { Hugar } \\
\text { et al. } .^{24}\end{array}$ & $\begin{array}{l}\text { Ensayo } \\
\text { Clínico }\end{array}$ & 60 & $\begin{array}{l}4 \text { a } 6 \\
\text { años }\end{array}$ & & 30 & & & & & & 100,0 & & 30 & & & & & & 100,0 \\
\hline S11 & $\begin{array}{l}\text { Jayam } \\
\text { et al. } .^{25}\end{array}$ & $\begin{array}{l}\text { Ensayo } \\
\text { Clínico }\end{array}$ & 100 & $\begin{array}{l}3 \text { a } 7 \\
\text { años }\end{array}$ & & 50 & & & & & & 100,0 & & 50 & & & & & & 100,0 \\
\hline $\mathrm{S} 12$ & $\begin{array}{l}\text { Yildirim } \\
\text { et al. }{ }^{26}\end{array}$ & $\begin{array}{l}\text { Ensayo } \\
\text { Clínico }\end{array}$ & 70 & $\begin{array}{l}5 \text { a } 9 \\
\text { años }\end{array}$ & & 35 & & & & & & 100,0 & & 35 & 1,0 & & & 1.0 & 1.1 & 96,9 \\
\hline S13 & $\begin{array}{l}\text { Biedma } \\
\text { et }^{\text {al. }}{ }^{6}\end{array}$ & $\begin{array}{l}\text { Ensayo } \\
\text { Clínico }\end{array}$ & 212 & $\begin{array}{r}2 \text { a } 10 \\
\text { años }\end{array}$ & & 138 & 1,2 & & 1,2 & & & 98,6 & & 74 & 1,2 & & 2,9 & & & 95,9 \\
\hline S14 & $\begin{array}{l}\text { Olatosi } \\
\text { et al. }{ }^{27}\end{array}$ & $\begin{array}{l}\text { Ensayo } \\
\text { Clínico }\end{array}$ & 50 & $\begin{array}{l}4 \text { a } 7 \\
\text { años }\end{array}$ & & 25 & & & & & & 100,0 & & 25 & & & 4,2 & & & 95,8 \\
\hline S15 & $\begin{array}{l}\text { Yildiz } \\
\text { et }_{\text {al. }}{ }^{18}\end{array}$ & $\begin{array}{l}\text { Ensayo } \\
\text { Clínico }\end{array}$ & 140 & $\begin{array}{l}5 \text { a } 9 \\
\text { años }\end{array}$ & & 41 & & & & 3,6 & & 96,4 & & 35 & & & & & & 100,0 \\
\hline & & & & & & & & & & Pror & edio & 99,0 & & & & & & Pron & edio & 98,3 \\
\hline
\end{tabular}

Leyenda

\begin{tabular}{|l|l|l|}
\hline Hallazgos & Sigla & \multicolumn{1}{|c|}{ Significado } \\
\hline \multirow{4}{*}{ Clínicos } & $\mathrm{AB}$ & Absceso \\
\cline { 2 - 3 } & $\mathrm{IG}$ & Inflamación Gingival \\
\cline { 2 - 3 } & $\mathrm{MP}$ & Movilidad Patológica \\
\cline { 2 - 3 } & $\mathrm{DP}$ & Dolor a la Percusión \\
\cline { 2 - 3 } & $\mathrm{DE}$ & Dolor Espontáneo \\
\hline
\end{tabular}


Tabla 7: Resultados de Eficacia Clínica entre el MTA e Hidróxido de Calcio obtenidos en la Extracción de Datos

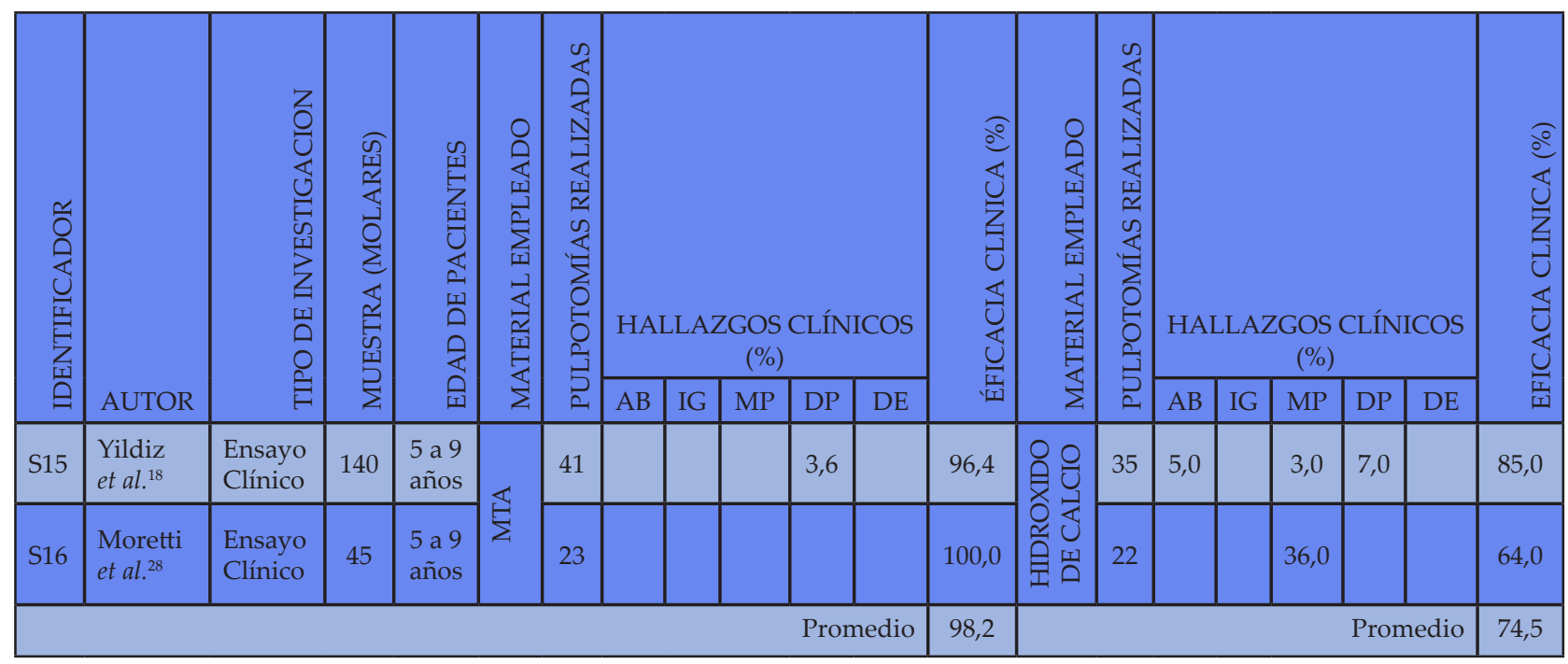

Leyenda

\begin{tabular}{|l|l|l|}
\hline Hallazgos & Sigla & \multicolumn{1}{|c|}{ Significado } \\
\hline \multirow{4}{*}{ Clínicos } & $\mathrm{AB}$ & Absceso \\
\cline { 2 - 3 } & $\mathrm{IG}$ & Inflamación Gingival \\
\cline { 2 - 3 } & $\mathrm{MP}$ & Movilidad Patológica \\
\cline { 2 - 3 } & $\mathrm{DP}$ & Dolor a la Percusión \\
\cline { 2 - 3 } & $\mathrm{DE}$ & Dolor Espontáneo \\
\hline
\end{tabular}

Reporte de Resultados

De la Tabla 5, que proyecta los resultados de eficacia clínica obtenidos respecto al cotejo del MTA y el Sulfato Férrico, se puede apreciar que utilizando el MTA en los estudios S1, S2 y S4 no existe evidencia de los hallazgos evaluados, por lo tanto, su éxito es $100 \%$. Sin embargo, en los artículos S3 y S15 se observó una eficacia clínica de 93,1 \% y 96,4 \% respectivamente. Al analizar los resultados del SF en S1 y S2 existe ausencia de hallazgos clínicos, por consiguiente, su efectividad es del $100 \%$, mientras que en S3, S4 y S15 su rango de eficacia es del 63,6 \% al 95,2\%.

En la Tabla 6, que expone los resultados de la comparación entre el MTA y el
Formocresol, el MTA presentó un $100 \%$ de eficacia en ocho estudios: S6, S7, S8, S9, S10, S11, S12 y S14; pero en S5, S13 y S15 se obtuvo del $94 \%$ al 98,6 \%. En este contexto, con la aplicación del FC solo seis estudios (S6, S7, S9, S10, S11 y S15) lograron un éxito del $100 \%$. Además, se observó en S5, S8, S12, S13 y S14 un rango de $94 \%$ al $98,8 \%$ de eficacia.

Respecto a la Tabla 7, en donde se evalúa el MTA con el $\mathrm{CH}$, se revela que en S15 utilizando $\mathrm{CH}$ se alcanzó un $85 \%$ mientras que el MTA recabó 96,4\%. También, se evidencia en S16 una eficacia clínica para el MTA de $100 \%$ y para CH de $64 \%$.

Finalmente, se concluye que en relación a los hallazgos clínicos evaluados la eficacia 
promedio del MTA en contraposición al SF es de $97,9 \%$ y $86,9 \%$ respectivamente, esto se correlaciona con los valores obtenidos del MTA frente al FC cuya ponderación es $99 \%$ contra un $98,3 \%$. Además, en la comparación del MTA con $\mathrm{CH}$ el primero logró un $98,2 \%$ y el segundo $74,5 \%$.

Considerando los porcentajes alcanzados por el MTA respecto a sus homogéneos se deduce que a nivel clínico este medicamento ofrece una mejor respuesta en pulpotomías. Se observó que la presencia de puentes de dentina y su capacidad de sellado le otorga mayor éxito al ser una barrera entre ambos compartimientos que permite mantener a la pulpa vital sana evitando la microfiltración y el fracaso del tratamiento por necrosis pulpar. Entonces, la eficacia clínica del MTA está basado en tres características: biocompatibilidad, $\mathrm{pH}$ básico y su elevada facultad al sellar la cámara pulpar.

\section{Discusión}

La revisión sistemática de los dieciséis artículos seleccionados demuestra que los hallazgos clínicos evaluados para el MTA en contraste con el FC, SF e CH confieren un resultado más favorable para el MTA con 98,4 \% de eficacia clínica en la realización de pulpotomías. A su vez, Yildiz et al. ${ }^{18}$ evidencian que su capacidad de sellado y al no mostrar signos de solubilidad le proporcionan mayor ventaja en el tratamiento.

Por su parte, De Vasconcelos et al. ${ }^{29}$ manifiestan que el pH alcalino del MTA posibilita sus efectos antibacterianos e incluso ayuda a la formación de tejidos duros, lo cual concuerda con la afirmación de Vallejo et al. ${ }^{30}$ quienes revelan que el $\mathrm{pH}$ es el responsable de la eficacia de sus propiedades biológicas garantizando la antisepsia y estimular la calcificación.

Si examinamos los datos obtenidos por Ansari et al. ${ }^{22}$ se deduce que el Formocresol posee una eficacia clínica de $98,8 \%$, resultados que son similares a los conseguidos en esta revisión. Sin embargo, en el estudio de Patchett et al. ${ }^{31}$ quedó demostrada la ineficacia del material al presentar mutación de células diploides humanas de la línea linfoblásticas en cultivos de células. También, Assed et al. ${ }^{5}$ exponen que los dientes tratados con FC podrían exfoliarse precozmente. En este contexto, González et al. ${ }^{32}$ señalan que al diluir el formocresol a 1:5 o disminuir el tiempo de aplicación podría reducir su toxicidad en pulpas vitales de dientes primarios, lo que ayudaría a prevenir el daño hacia las capas más profundas.

En otro ámbito, el MTA y $\mathrm{CH}$ estimulan la presencia de puentes de dentina, por su biocompatibilidad y alcalinidad ${ }^{19}$; no obstante, en esta investigación el Hidróxido de Calcio no supera el $75 \%$ de efectividad. A diferencia del MTA, el CH puede ocasionar microfiltración bacteriana a nivel de la furca o de la restauración coronal, factor que se relaciona al fracaso clínico del tratamiento. ${ }^{33}$

Según Srinivasan et al. ${ }^{4}$, el $\mathrm{CH}$ ha sido muy aplicado en el procedimiento de pulpotomía, pero los porcentajes de eficacia son limitados debido al desarrollo de inflamación crónica de la pulpa. Además, Castillo et al. ${ }^{34}$ manifiestan como efecto secundario la presencia 
de reabsorción interna al producirse la formación del coágulo de sangre entre el material y el tejido pulpar.

Por otra parte, Zaror et al. ${ }^{3}$ proponen al SF como alternativa favorable en las pulpotomías por la rapidez en la aplicación y su bajo costo; sin embargo, en esta revisión alcanzó el 74,5 \% de éxito. En consecuencia, las características señaladas no justificarían su uso en Odontopediatría, por lo que surge la necesidad de ejecutar mayor cantidad de ensayos clínicos para evaluar su eficacia.

\section{Conclusiones}

El MTA logró un porcentaje de eficacia clínica en pulpotomías del $99 \%$ en contraste al 98,3\% del formocresol. Además, recabó una efectividad de $97,9 \%$ frente al $86,9 \%$ del sulfato férrico y finalmente, el MTA alcanzó un 98,2 \% de eficacia ante un 74,5\% del hidróxido de calcio. Por consiguiente, se deduce que el MTA obtuvo mayor eficacia por su biocompatibilidad, $\mathrm{pH}$ básico y su elevada facultad al sellar la cámara pulpar; por lo tanto, puede emplearse como un material seguro en pulpotomías de dientes primarios.

Dentro de las limitaciones de este trabajo se encuentra la presencia de pocos ensayos clínicos entre el MTA y el CH. Además, algunas investigaciones exponen datos globales, es decir, que no especifican el éxito de los materiales usados de acuerdo a cada hallazgo clínico, lo que reduce la selección de artículos.

\section{Referencias bibliográficas}

1. Odabas M, Bodur H, Baris E, Demir C. Clinical, Radiographic, and Histopathologic Evaluation of Nd: YAG Laser Pulpotomy on Human Primary Teeth. J Endod [Internet]. 2007 [Consultado 2019 Jun 10]; 33: 415-21. Disponible en: https://www.jendodon.com/article/S0099-2399(06)01221-0/fulltext

2. IARC Classifies Formaldehyde As Carcinogenic To Humans. IARC Monogr Eval Carcinog Risks Hum [Internet]. 2006 [Consultado 2019 May 10]; 62:217-375 Disponible en: https://monographs.iarc.fr/wpcontent/uploads/2018/06/mono88.pdf

3. Zaror C, Vergara C, Díaz J, Aracena D. Pulpotomías con sulfato férrico y MTA en dientes primarios: serie de casos. Int. J. Odontostomat [Internet]. 2011 [Consultado 2019 Jun 2]; 5(1):77-82. Disponible en: https:// scielo.conicyt.cl/scielo.php?script=sci_arttext\&pid=S0718-381X2011000100012

4. Srinivasan V, Patchett CL, Waterhouse PJ. Is there life after Buckley's Formocresol? Part I - A narrative review of alternative interventions and materials. Int J Paed Dent [Internet]. 2006 [Consultado 2019 Jun 10]; 16: 117-27. Disponible en: https://www.ncbi.nlm.nih.gov/pubmed/16430526

5. Assed S, Assed L, Nelson-Filho P. Pulpotomía en dientes temporales y permanentes jóvenes. En: Assed L, editor. Tratado de Odontopediatría. 1 ed. Colombia: Amolca; 2008. p. 571-611.

6. Biedma M, Solano B, García F, Mendoza A, Iglesias A. Clinical and radiographic evaluation of white MTA versus formocresol Pulpotomy: a 48-month follow-up study. Am J Dent [Internet]. 2017 [Consultado 2019 Jun 2]; 30(3):131-136. Disponible en: https://www.ncbi.nlm.nih.gov/pubmed/29178757

7. Valdivieso M, Huamán M. Diagnóstico y tratamiento pulpar. En: Castillo R Perona G, Kanashiro C, Perea M, Silva-Esteves F, editores. Estomatología Pediátrica. 1 ed. España:Ripano “Editorial Médico"; 2011. p. 174-99.

8. Rodríguez P; Bolaños V. Propiedades y Usos en Odontopediatria del MTA (Agregado de Trióxido Mineral) Odovtos - International Journal of Dental Sciences [Internet]. 2011 [Consultado 2019 Jun 2]; 13, 2011, pp. 65-70 Disponible en: https://revistas.ucr.ac.cr/index.php/Odontos/article/view/4772 
9. Aguado J, Cruz I,Maroto M, Barbería E. (2009). Posibilidades terapéuticas del Agregado Trióxido Mineral (MTA) en odontopediatría. JADA [Internet]. 2009 [Consultado 2019 Jun 2]; 4. 185-193. Disponible en: https://www.researchgate.net/publication/257920006_Posibilidades_terapeuticas_del_Agregado_ Trioxido_Mineral_MTA_en_odontopediatria

10. Hincapié S, Valerio AL. Biodentine: una nueva propuesta en terapia pulpar. Univ Odontol. 2015 [Consultado 2019 May 10]; 34(73): 69-76. Disponible en: https://revistas.javeriana.edu.co/index.php/ revUnivOdontologica/article/view/16040

11. Caicedo R, Abbott P, Alongi D, Alarcon M. Clinical, radiographic and histological analysis of the effects of mineral trioxide aggregate used in direct pulp capping and pulpotomies of primary teeth. Austr Dent J [Internet]. 2006 [Consultado 2019 Jun 14]; 51(4): 297-305. Disponible en: https://www.ncbi.nlm.nih.gov/ pubmed/17256303

12. Higgins JPT, Green S (editors). Cochrane Handbook for Systematic Reviews of Interventions Version 5.1.0 The Cochrane Collaboration [Internet]. 2011 [Consultado 2019 May 14]. Disponible en: https://es.cochrane. org/sites/es.cochrane.org/files/public/uploads/manual_cochrane_510_web.pdf

13. Kitchenham B. Procedures for Performing Systematic Reviews. Department of Computer Science, Keele University Technical Report [Internet]. 2004 [Consultado 2019 May 12]; 33. Disponible en: www.inf.ufsc. br/ aldo.vw/kitchenham.pdf

14. Kitchenham B, Brereton P, Budgen D, Turner M, Bailey J, Linkman S. Systematic literature reviews in software engineering-A systematic literature review. Information and Software Technology [Internet]. 2009 [Consultado 2019 May 12]; 51. 7-15. Disponible en: https://www.sciencedirect.com/science/article/ abs/pii/S0950584908001390

15. Junqueira M, Cunha N, Caixeta, Marques N, Oliveira T, Moretti A, Cosme L, Sakai V. Clinical, Radiographic and Histological Evaluation of Primary Teeth Pulpotomy Using MTA And Ferric Sulfate. Braz. Dent. J [Internet]. 2018 [Consultado 2019 Jun 2]; 29(2): 159-165. Disponible en: http://www.scielo.br/scielo. php?script=sci_arttext\&pid=S0103-64402018000200159\&lng=en.

16. Guven Y, Aksakal S, Avcu N, Unsal G, Tuna E, Aktoren O. Success Rates of Pulpotomies in Primary Molars Using Calcium Silicate-Based Materials: A Randomized Control Trial. Biomed Res Int [Internet]. 2017 [Consultado 2019 Jun 2]; 2017. Disponible en: https://www.ncbi.nlm.nih.gov/pubmed/29226134

17. Goyal P, Pandit I, Gugnani N, Gupta M, Goel R, Gambhir R. Clinical and radiographic comparison of various medicaments used for pulpotomy in primary molars: A randomized clinical trial. Eur J Dent [Internet]. 2016 [Consultado 2019 Jun 2]; 10:315-20. Disponible en: https://www.ncbi.nlm.nih.gov/ pubmed/27403046

18. Yildiz E, Tosun G. Evaluation of formocresol, calcium hydroxide, ferric sulfate, and MTA primary molar pulpotomies. Eur J Dent [Internet]. 2014 [Consultado 2019 Jun 2]; 8:234-40. Disponible en: https://www. ncbi.nlm.nih.gov/pubmed/24966776

19. Biondi A, Cortese S, Ortolani A. Pulpotomías en molares primarios evaluación clínica, radiográfica de formocresol o trióxido mineral agregado. Revista de la Facultad de Odontología- Repositorio (UBA) [Internet]. 2008 [Consultado 2019 Jun 2]; 23:54-55. Disponible en: http://od.odontologia.uba.ar/ revista/2008vol23num54-55/art2.pdf

20. Noorollahian H. Comparison of mineral trioxide aggregate and formocresol as pulp medicaments for pulpotomies in primary molars. Br Dent J [Internet]. 2008 [Consultado 2019 Jun 2]; 204(11). Disponible en: https://www.ncbi.nlm.nih.gov/pubmed/18425074

21. Hugar S, Deshpande S. Comparative investigation of clinical/radiographical signs of mineral trioxide aggregate and formocresol on pulpotomized primary molars. Contemporary clinical dentistry [Internet]. 2010 [Consultado 2019 Jun 2]; 1(3), 146-151. Disponible en: https://www.ncbi.nlm.nih.gov/pmc/articles/ PMC3220100/

22. Ansari G, Ranjpour M. Mineral trioxide aggregate and formocresol pulpotomy of primary teeth: a 2-year follow- up. International Endodontic Journal [Internet]. 2010 [Consultado 2019 Jun 2]; 43(5):413-8. Disponible en: https://www.ncbi.nlm.nih.gov/pubmed/20518934

23. Godhi B, Sood P, Sharma A. Effects of mineral trioxide aggregate and formocresol on vital pulp after pulpotomy of primary molars: An in vivo study. Contemporary clinical dentistry [Internet]. 2011 [Consultado 2019 Jun 2]; 2(4), 296- 301. Disponible en: https://www.ncbi.nlm.nih.gov/pubmed/22346156

24. Hugar S, Reddy R, Deshpande S, Shigli A, Gokhale N, Hugar S. In vivo Comparative Evaluation of Mineral Trioxide Aggregate and Formocresol Pulpotomy in Primary Molars: A 60-month Follow-up Study. Contemporary clinical dentistry [Internet]. 2017 [Consultado 2019 Jun 2]; 8(1), 122-127. Disponible en: https://www.ncbi.nlm.nih.gov/pmc/articles/PMC5426144/ 
25. Jayam C, Mitra M, Mishra J, Bhattacharya B, Jana B. Evaluation and comparison of white mineral trioxide aggregate and formocresol medicaments in primary tooth pulpotomy: clinical and radiographic study. J Indian Soc Pedod Prev Dent [Internet]. 2014 [Consultado 2019 Jun 2]; 32(1):13-8. Disponible en: https:// www.ncbi.nlm.nih.gov/pubmed/24531596

26. Yildirim C, Basak F, Akgun O, Polat G, Altun, C. Clinical and radiographic evaluation of the effectiveness of formocresol, mineral trioxide aggregate, portland cement, and enamel matrix derivative in primary teeth pulpotomies: a two year follow-up. J Clin Pediatr Dent [Internet]. 2016 [Consultado 2019 Jun 2]; 40(1):14-20. Disponible en: https://www.ncbi.nlm.nih.gov/pubmed/26696101

27. Olatosi $\mathrm{O}$, Sote E, Orenuga O. Effect of mineral trioxide aggregate and formocresol pulpotomy on vital primary teeth: a clinical and radiographic study. Niger J Clin Pract [Internet]. 2015 [Consultado 2019 Jun 2]; 18(2):292-6. Disponible en: https://www.ncbi.nlm.nih.gov/pubmed/25666010

28. Moretti A, Sakai V, Oliveira T, Fornetti A, Santos C, Machado M, Abdo R. The effectiveness of mineral trioxide aggregate, calcium hydroxide and formocresol for pulpotomies in primary teeth. Int Endod J [Internet.] 2008 [Consultado 2019 Jun 2]; 41(7):547-55. Disponible en: https://www.ncbi.nlm.nih.gov/ pubmed/18479381

29. De Vasconcelos B , Bernardes R , Cruz S, Duarte M , Padilha M , Bernardineli N , Garcia R , Bramante C, de Moraes I . Evaluation of $\mathrm{pH}$ and calcium ion release of new root-end filling materials. Oral Surg Oral Med Oral Pathol Oral Radiol Endod. [Internet]. 2009 [Consultado 2019 Jun 2]; 108(1):135-9. Disponible en: https://www.ncbi.nlm.nih.gov/pubmed/19451009

30. Vallejo E, Rosales J. Materiales dentales. En: Boj JR. Odontopediatría. La evolución del niño al adulto joven. Madrid: Ripano editorial médica; 2011. p. 195-210.

31. Patchett CL, Srinivasan V, Waterhouse PJ. Is there life after Buckley's formocresol? Part II - Development of a protocol for the management of extensive caries in the primary molar. Int J Paed Dent [Internet]. 2006 [Consultado 2019 Jun 2]; 16: 199-206 Disponible en: https://www.ncbi.nlm.nih.gov/pubmed/16643542

32. Gonzalez Rodriguez E., Ruiz Linares M. Diagnóstico y tratamiento en dentición temporal. En: Boj JR, Catalá M, García-Ballesta C, Mendoza A. Odontopediatría. 1aㅡ ed. Barcelona: Masson; 2004 p.173-183.

33. Isassi H, Mendoza M, Padilla C. Estudio clínico de pulpotomía con agregado trióxido mineral en 58 molares primarios. Medigraphic [Internet]. 2012 [Consultado 2019 Jun 10]; Vol. 37. Disponible en: https:// www.medigraphic.com/pdfs/oral/ora-2011/ora1137g.pdf

34. Castillo R, Perona G, Kanashiro C, Perea M, Silva F. ESTOMATOLOGÍA PEDIÁTRICA, edit. RIPANO Madrid 2010; (174-191).

Recibido: $26 / 01 / 2020$

Aceptado: 27/05/2020

Correspondencia: Sol Gabriela Holguín García, correo: sol.holguin89@hotmail.com 